\title{
Visual Acuity Before and After Treatment in Patients with Chemical Injuries at the National Eye Center, Cicendo Eye Hospital, Bandung from 2010 to 2011
}

\author{
Endi Pramudya Laksana, ${ }^{1}$ Nina Ratnaningsih, ${ }^{2}$ Reni Farenia $S^{3}$ \\ ${ }^{1}$ Faculty of Medicine Universitas Padjadjaran, ${ }^{2}$ Department of Ophthalmology, Faculty of \\ Medicine, Universitas Padjadjaran/National Eye Center, Cicendo Eye Hospital Bandung, \\ ${ }^{3}$ Department of Physiology Faculty of Medicine Universitas Padjadjaran
}

\begin{abstract}
Background: Chemical trauma is one of the emergency cases in ophthalmology since it can lead to severe, permanent blindness if not immediately treated. This study aimed to reveal pre- and post-therapy visual acuity on patients with chemical trauma at theNational Eye Center, Cicendo Eye Hospital, Bandung.

Methods: This study was performed on 40 patients' medical records from the National Eye Center, Cicendo Eye Hospital Bandung from January 2010 to January 2011 as secondary data, using the descriptive retrospective method. The data were divided into two groups: acid and alkali trauma. The collected data were analyzed and presented in tables.

Results: Chemical trauma cases were mostly caused by caustic soda $(17 / 40)$ whereas the least were caused by vinegar (3/40) and commonly occurred on adult patients in the right eye on alkali trauma. Patients with chemical trauma generally had normal eyesight before therapy.The number of patients with acid and alkali trauma who experienced improved eyesight after therapy alkali were similar (3 patients) while worsening symptoms occurred in one patient with alkali chemical trauma from mild to medium low vision.

Conclusions: There are some improvements of visual acuity after treatment in patients with chemical injuries. [AMJ.2015;2(3):387-90]
\end{abstract}

Keywords: Acid trauma, alkali trauma, visual acuity

\section{Introduction}

The eye function can be disrupted or even permanently vanished if exposed to foreign objects, including acid or alkali chemical

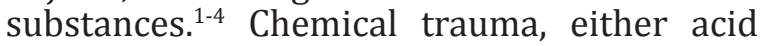
or alkali, on eyes can be an emergency case in ophthalmology since it can cause severe and permanent blindness if not immediately treated. ${ }^{2,5-7}$

According to data of the Centers for Disease Control and Prevention (CDC) ${ }^{8}$ in 1998, chemical trauma includes $84 \%$ eye trauma, and $80 \%$ of chemical trauma incidents commonly occur in workplaces. The frequency ratio of acid and alkali chemical trauma can vary, from 0.25 to 1 . Men have 4 times the risk of getting chemical trauma than women.

Notable main causes of acid trauma are sulfuric acid (H2SO4) and hydrochloric acid
(HCI) often used as substances for household cleaning for floors and ceramics. ${ }^{9}$ Acids tend to cause less severe damage since cornea proteins will react with acids, causing precipitations on cornea surface, thus preventing chemical substances from entering deeper into eye tissues. Nevertheless, strong acids such as sulfuric acids can cause severe damages, since severe acid trauma can damage the ciliary body, thus lowering the ascorbate level in eye fluids and cornea. ${ }^{2,7}$

Alkali trauma is commonly more severe than acid trauma since alkali substances have two characteristics: hydrophilic and lipophilic. Both can quickly cause the penetration of the chemical into the anterior segment along with collagen hydration, malignant fibrial changes and trabecular changes. ${ }^{5}$ These conditions can cause a rapid and significant change in intraocular pressure second to a rapid rise in aqueous humor. ${ }^{5}$ Alkali trauma can cause light

Correspondence: Endi Pramudya Laksana, Faculty of Medicine, Universitas Padjadjaran, Jalan Raya Bandung-Sumedang Km.21, Jatinangor, Sumedang, Indonesia, Phone: +6287737375353 Email: endi_pramudya@yahoo.com 
or severe irritation on eyes and can cause emergency cases that can lead to blindness. ${ }^{5}$ Blindness can occur if the patient is receiving delayed therapy, especially after being exposed to chemical trauma.

The proper therapy to treat cyhemical trauma is by providing irrigation to the exposed eye by using sterile buffer solution such as Ringer's lactate or normal saline. According to studies by the American Science in the United States6, approximately 70\% of 50,000 patients with chemical trauma gained $20 / 20$ vision after they were given the irrigation therapy.

A lot of people consider exposures to chemical substances on the eyes as normal cases, and they only rinse their eyes with water after exposures. Such notions are the result of lack of understanding among people about the effects resulted from chemical trauma, either acid or alkali, on the eyes. Furthermore, eye protections in workplaces have often been neglected, either personally or institutionally. ${ }^{9}$ Eye protection is actually the company's responsibilty. Neverhteless, it is advised to use our own eye protections in order to maintain protections for our eyes.

Chemical trauma which can cause permanent blindness, as well as the fact that there have been no adequate scientific data on visual acuity on patients with chemical trauma in Bandung became the background of this study in revealing visual acuity, both preand post-therapy, on patients with chemical trauma at the National Eye, Cicendo Eye Hospital, Bandung,over the period of January 2010-January 2011.

\section{Methods}

This study used the retrospective descriptive method, using cases of patients with chemical trauma who came to the Emergency Room of theNational Eye Center, Cicendo Eye Hospital, Bandung, from January 2010 to January 2011. Secondary data were retrieved from medical
Table 1 Distribution of Chemical Trauma Based on Chemical Substances

\begin{tabular}{lc}
\hline Chemical Substance & Frequency \\
\hline Caustic Soda $(\mathrm{NaOH})$ & 17 \\
Superglue & 5 \\
Vinegar & 3 \\
Albothyl & 4 \\
Battery fluid & 11 \\
\hline
\end{tabular}

records at Cicendo Eye Hospital Bandung. The subject of this study were the medical records of patients with chemical trauma at the Emergency Room of Cicendo Eye Hospital, Bandung which fulfilled both the inclusion and exclusion criteria.

The inclusion criteria were all patients with chemical trauma who were treated at Cicendo Eye Hospital, Bandung, from 2010 to 2011. While the exclusion criteria were medical records without pre- and post-therapy visual acuity data. Data were retrieved from Cicendo Eye Hospital, Bandung during September to November 2012. Data processing was conducted during August-December 2012, using the Microsoft Excel software program to reveal frequency data. The results were presented in tables.

\section{Results}

Chemical trauma cases were mostly caused by caustic soda $(17 / 40)$, whereas the least were caused by vinegar $(3 / 40)$ (Table 1$)$.

Table 2 shows that acid and alkali chemical trauma cases based on age category of the patients commonly occurred on adult patients. Meanwhile, based on location, the exposed mostly happened in the right eye on alkali trauma (Table 3).

Table 4 shows that patients with chemical trauma generally had normal eyesight before therapy. Approximately 8/12 patients with

Table 2 Distribution of Chemical Trauma Based on Age Category

\begin{tabular}{lcc}
\hline \multicolumn{1}{c}{ Age } & Acid & Alkali \\
\cline { 2 - 3 } & Frequency & Frequency \\
\hline Children (5-11 years old) & 2 & 0 \\
Teen (12-17 years old) & 2 & 1 \\
Adult (18-40 years old) & 6 & 24 \\
Elderly (41-65 years old) & 2 & 3 \\
\hline
\end{tabular}


Table 3 Distribution of Chemical Trauma Based on Location of Eyes Exposed to Chemical Substances

\begin{tabular}{lcc}
\hline \multirow{2}{*}{ Location } & Acid & Alkali \\
\cline { 2 - 3 } & Frequency & Frequency \\
\hline Right Eye & 5 & 17 \\
Left Eye & 5 & 9 \\
Left and Right Eyes & 2 & 2 \\
\hline
\end{tabular}

acid chemical trauma had normal eyesight, and only $3 / 12$ had medium low vision. There were no patients with blind eyesight. On the other hand in alkali, approximately 20/28 patients with alkali chemical trauma had normal eyesight and only $2 / 28$ had medium low vision. The number of patients who experienced improved eyesight after therapy was 3 patients with acid and alkali trauma while, worsening symptoms occurred on one patient with alkali chemical trauma from mild to medium LV.

\section{Discussions}

Caustic soda was the most frequent cause of chemical trauma in this study. It is a alkali chemical substance often used in thegarment industry for enhancing clothing colors. Chemical trauma cases caused by caustic soda occurred more frequently than other chemical substances, since many laborers were coloring clothing without wearing eye protection.

Other studies revealed that chemical substances that mostly caused chemical trauma were caustic soda and ammonia., ${ }^{2,3}$ Both of these chemical substances are the most dangerous ones as they can cause severe complications on theeyes which is also related to the occupations of patients with chemical trauma. The frequency of laborers in thegarment industry was more thanin any other occupations related to chemical substances. ${ }^{3,4}$

Table 2 shows that both acid and alkali chemical trauma cases based on age category of the patients commonly occurred on adult patients. This was in accordance with the epidemiology data where chemical trauma cases mostly occur on adult patients due to their occupations.

According to the epidemiology data, around $60 \%$ of such cases occured in their workplaces, $30 \%$ in their house, and $10 \%$ in other places. ${ }^{2} 80 \%$ of cases occur adult patients whose occupations were related to chemical substances. ${ }^{10}$

Table 3 shows that most of the distributions of chemical trauma on eyes based on the location of eyes exposed to chemical substances were as follows: 5 patients (42\%) on the right eye, 5 patients $(42 \%)$ on the left eye, and 2 patients $(16 \%)$ on both eyes.

Table 3 also shows that most of the distributions of chemical trauma cases on patients basedon the location of the eye exposed to chemical substances at Cicendo Eye Hospital, Bandung, were on the right eye. This result was in line with other studies that also reported that chemical trauma on both eyes (bilateral) were rare. ${ }^{3}$

This study reported that improvements occurred in both chemical trauma. There were some LV improvements both in acid and alkali trauma but worsening symptoms also happened but in smaller number. According

Table 4 Pre and Post Therapy Visual Acuity

\begin{tabular}{lcccc}
\hline \multirow{2}{*}{ Visual Acuity } & \multicolumn{2}{c}{ Acid } & \multicolumn{2}{c}{ Alkali } \\
\cline { 2 - 5 } & Pre Therapy & Post Therapy & Pre Therapy & Post Therapy \\
\hline Normal $(\geq 0.8)$ & 8 & 9 & 20 & 23 \\
Mild LV (0.3-0.63) & 1 & 3 & 7 & 3 \\
Medium LV (0.125-0.25) & 3 & 0 & 1 & 2 \\
Severe LV (0.05-0.10) / & 0 & 0 & 0 & 0 \\
NLP & & & & \\
\hline
\end{tabular}

*LV: Low Visio; NLP: No Light Perception 
to other studies, visual acuity of patients with chemical trauma given with therapy will 95\% improve, $15 \%$ improve after 10-14 days, and $80 \%$ improve on thefifth to eighth week after therapy. ${ }^{3}$

It was noted that patients with chemical trauma who had not yet been given treatments or therapy stated they experienced blurred visions, with red, painful eyes. However, if they had previously performed simple treatments such as rinsing the exposed eye(s) with water or ringer lactate, their vision improved to normal and there were no further complications on exposed eye(s). ${ }^{3}$ Such treatments only serve as temporary treatments. If patients suffers from chemical trauma on their eyes, it is highly recommended for them to visit the nearest hospital for therapy and to avoid further complications. ${ }^{12}$

The time range between chemical exposure and irrigation treatment is the most important factor in the improvement of visual acuity. There is one worsened symptom in alkali chemical trauma that may be caused by the longer time range between chemical exposure and irrigation treatment, thus the chemical substance have penetrated into deeper eye tissues and caused severe damage on their eye. ${ }^{2,11}$

The study concludes that the number of sample patients with acid chemical trauma who experienced improved visual acuity after therapy is similar in quantity, but there is a worsening symptom in alkali chemical trauma since alkali can quickly cause the penetration of the chemical into the anterior segment along with collagen hydration, malignant fibrial changes and trabecular changes. ${ }^{5}$ The study has several limitations by not including the detailed type of acid and alkali solution, time ranging between exposure and treatment, type of treatment, and the time of post treatment determination of visual acuity that may influence the improvement of visual acuity. Furthermore, there should be counseling activities for people concerning causes, effects, and treatments of chemical trauma, thus they can directly obtain first aid and have their visual acuity improve to normal immediately after having their eye(s) exposed to chemical substances.

\section{References}

1. Lecuona K. Assessing and managing eye injuries. Community Eye Health Journal. 2005;18(55):102-16.

2. Venkatesh R, Trivedi HL. Ocular traumachemical injuries. Bombay Hospital Journal. 2009;51(2):215-21.

3. Trudo EW Jr, Rimm W. Chemical injuries of the eye. In: Thach $A B$, editor. Ophthalmic care of the combat casuality. Washington: Office of The Surgeon General United States Army; 2003. p. 115-35.

4. Pons J. Eye trauma. CME. 2011;29(2):66-8.

5. Kosoko A, Vu Q, Kosoko-Lasaki O. Chemical ocular burns: a case review. Am J Clin Med. 2009;6(3):41-9.

6. Sesha $\mathrm{H}$, Hashem $\mathrm{H}$, Liang L, Ramzy $\mathrm{M}$, Zaki A. Amniotic membrane extract for acute ocular chemical burns. J Am Sci. 2010;6(11):427-33.

7. Ilyas S. Ilmu penyakit mata. Jakarta: Balai Penerbit FKUI; 1997:p.76-8.

8. Centers for Disease Control and Prevention. Work-related eye injuries. 2010. (Cited 2012 August 18). Available from: http:// www.cdc.gov/features/dswork PlaceEye/

9. Centers for Disease Control and Prevention. Workplace safety \& health topics: eye safety. 2007 (Cited 2012 August 18). Available from: www.cdc.gov/ niosh/topics/eye.

10. Fish R, Davidson RS. Management of ocular thermal and chemical injuries, including amniotic membrane therapy. Curr Opin Ophthalmol. 2010;21(4):317-21.

11. Kobayashi A, Shirao Y, Yoshita T, Yagami K, Segawa Y, Kawasaki K, et al. Temporary amniotic membrane patching for acute chemical burns. Eye (Lond). 2003;17(2):149-58.

12. Ulakhan 00, K? klü G, Firat E. Nonpreserved human amniotic membrane transplantation in acute and chronic chemical eye injuries. Cornea. 2002;21(2):169-72. 